\title{
The Design of a Rule Base for an e-Learning Recommendation System Base on Multiple Intelligences
}

\author{
T. Kaewkiriya, N. Utakrit, and M. Tiantong
}

\begin{abstract}
The problem of e-learning systems, learners are given learning contents that do not match individual aptitudes. This paper aims to design a rule base for recommendations focusing on e-learning and learning profiles which are based on multiple intelligences. Design of the rule base was divided into four sections as follows. The first section covered a survey of the variables. Second section was creation of the questionnaire. Third section was a survey of the student sample groups. The last section was an analysis of data generated from the results of the survey. The process of selection for the rule base was undertaken by comparing the performance of the following algorithms 1) ID3 algorithm 2) C4.5 algorithm 3) NBTree algorithm 4) Naïve Bayes algorithm 5) Bayes Net algorithm. The C4.5 algorithm had the highest percentage of prediction. Percentage of prediction from the $\mathrm{C} 4.5$ algorithm equaled 83.436\%.
\end{abstract}

Index Terms-E-learning, recommendation system, data mining, multiple intelligence.

\section{INTRODUCTION}

In traditional teaching, teachers determine the environment of all classes and learning methods. The current system of learning and teaching has evolved from the original, for example the e-learning system [1], distance learning [2]. The condition of learning and teaching today provides learners with opportunities to learn by themselves [3], teachers are only a guide. Generally, each learner has different abilities or aptitudes. Therefore, iflearners are not equally skilled in the class, the learning performance becomes unbalanced and some learners might fall behind.

Past research has offered various types of learning and teaching methods, such as learning and teaching by brainstorming [4]. Learning and teaching by brainstorming means that learners will participate in problem solving in the class. If learners brainstorm, the more opportunity to solve problems arises. The advantage of learning and teaching in this way helps groups of learners to solve problems faster than normal learning and teaching. The limitation of this method is that learners do not gain learning contents according to the individual's aptitude.

For this research [5] learning and teaching is a project base learning method (Project Base Learning) which focuses on every learner. From this learning and teaching method, learners can learn from the project at their own speed. The

Manuscript received August 29, 2014; revised October 30, 2014.

The authors are with the Faculty of Information Technology, King Mongkut's University of Technology North Bangkok, Bangkok, Thailand (e-mail: tkaewkiriya@gmail.com, Nattavee@kmutnb.ac.th, Monchai@kmutnb.ac.th). project is divided into appropriate groups of learners and learning contents. But the limitation occurs when learners are given contents that do not match their capabilities.

Researches [6]-[8] also use problem base learning (Problem Base Learning). This learning and teaching method focuses on learners by defining problems and solving them. This learning and teaching method is suitable for learners who are experts in analysis. But the restriction remains the same that learners do not get the lesson according to the individual's aptitude. Research [9] focuses on learning and teaching by emphasizing on learning activities base on Multiple Intelligence. This research focused on learning activities. But again was limited as individual's aptitudes were not catered for. In addition, [10] proposed learning activities by using Multiple Intelligences in the environment of e-learning. But the research focused on only learning activities and failed to consider learners with problems during study.

In addition, there is a form of learning and teaching that emphasizes learners as the center (Student Center Learning) [11]. This learning and teaching method focuses on the role of learners. Teachers are responsible for providing only a guide to learners. In the case that learners have problems and cannot solve them, teachers have a duty to assist and guide the learner to study further.

In addition, a group of researchers have used this technology to develop games with multimedia in order to help learning and teaching. Research [12], [13] are learning and teaching methods which use games to display learning material. This learning and teaching method is suitable for learners who enjoy playing games because when they play the game, they are learning at the same time. However, this application is suitable for only a small group of learners.

From the research mentioned above, learning and teaching by employing types of project base learning, problem base learning, activity base learning, game base learning and student center learning were found to be limited. In each method, learners are given learning contents that do not match individual aptitudes. Thus, this research presents the design of a rule base for e-learning recommendation. Rule bases are rules which come from the data analysis of learners. This research consists of 4 parts; First section, introduction of previous work. Second section covers a presentation of the background for this research. Third section, describes the concept of framework. Lastly, the proposed design of the rule base for learner recommendation.

\section{PREVIOUS WORK}

Research [14] presented the concept design as a model to 
guide e-learning learners according to the analysis of VARK base. The design of this model can guide learning contents of e-learning that match the aptitude of each learner. The aptitude of each learner can be analyzed according to the theory of VARK Model [15].VARK consists of visual techniques, which means visual learning, such as graphs, charts, flowcharts. Aural means learning though speech and dispute. Read/write means learning though reading and writing. Kinesthetic means learning from touching, testing and trail. Nevertheless, research [14] is still limited as the aptitudes of learners are not only 4 aspects. The aptitudes of learners have several aspects, such as Multiple Intelligence theory (MI) [16]. So, the guide according to VARK does not cover the aptitude of all learners.

Research [17] is about the learning activities based on analysis of Multiple Intelligences only. So, it does not cover the components of learning and teaching systems. In addition, research [18] also presented e-learning lessons by focusing on the contents but not on learners. So, learners with different aptitudes will be given the same contents and performance will not be as good as it should. Research [19] found the way to split learners according to their learning styles and learning management in relation to learners by trials with computer programming subjects. However, learners still get the same learning content, although there are separations of students group according to the type of learning.

Research [20] found that the creation of learning path of each learner and offered learning contents to learners. The creation of learning path of learner was from the history of users (Log file). In addition, it also adjusted learners' learning to be appropriate. However, this research is still limited as learners were given the same learning contents. Consequently, the performances of learners were not as good as it should be.

\section{BACKGROUND}

The theory of Multiple Intelligent [16] presents that each learner has different aptitudes which can be divided into cognitive aptitudes of humans by 9 types; 1) Verbal / Linguistic Intelligence, 2) Logical / Mathematical Intelligence, 3) Musical- Rhythmic Intelligence, 4) Body / Kinesthetic Intelligence, 5) Visual / Spatial Intelligence, 6) Interpersonal Intelligence, 7) Intrapersonal Intelligence, 8) Naturalist Intelligence, 9) Existential Intelligence. From 9 intelligences, each learner can have different dominant intellectual parts. The most important aspect is that from more dominant development, the result reflects individual progress. In addition, each learner may have more than one dominant area of intelligence. The Multiple Intelligence model is shown in Fig. 1.

The Multiple Intelligence theory is divided into 3 groups. 1) Analytic group, this group focuses on analysis and the thinking processes. The analytic group consists of 3 parts; Logical-mathematic intelligence, Musical intelligence, Naturalist intelligence. 2) Introspective group, this group focuses on imagination and understanding. The introspective group consists of 3 parts; Intrapersonal intelligence, spatial intelligence, and Existential intelligence. 3) Interactive group, this group focuses on communication and interactive. The interactive group consists of 3 parts; Linguistic intelligence, Interpersonal intelligence, and kinesthetic intelligence.

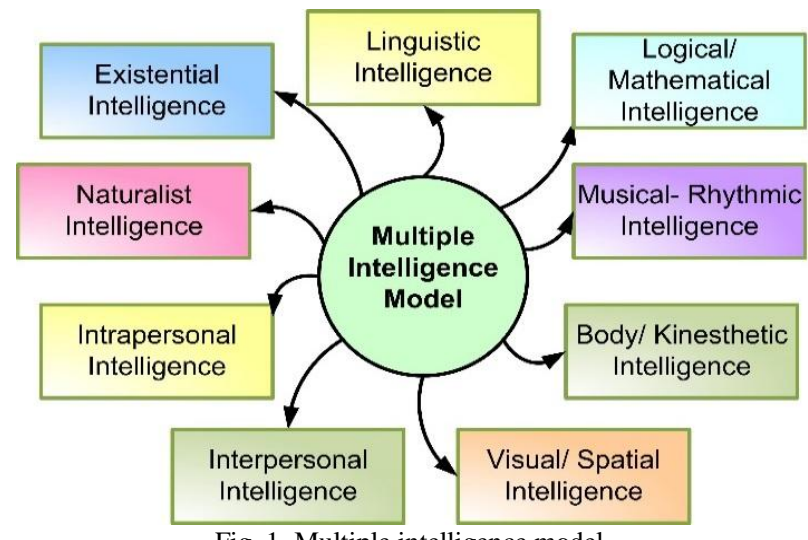

Fig. 1. Multiple intelligence model.

Fig. 1 shows the Multiple Intelligence model. This research uses the principle of Multiple Intelligence to design a rule base for the e-learning recommendation system. The design of the rule base is just one of five modules in the framework which is based on the Multiple Intelligence principle (see Fig. 2).

\section{CONCEPTUAL FRAMEWORK}

Fig. 2 shows the framework of an adaptive e-learning guidance system which consists of 5 modules [21], [22].

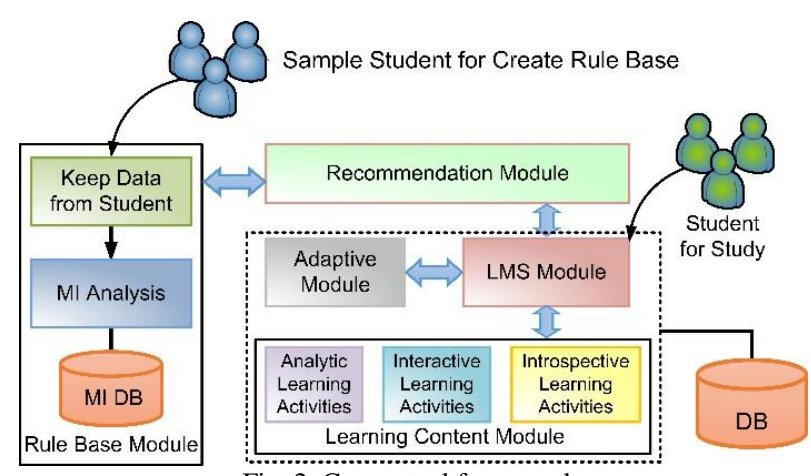

Fig. 2. Conceptual framework.

\section{A. Rule Base Module}

The Rule base module is the module that keeps the learning styles of learners according to their aptitude of Multiple Intelligences.

\section{B. Recommendation Module}

The recommendation module is the module that introduces learners to the type of learning content learners should get, such as Analytic Content, Introspective Content, or Interactive Content.

\section{LMS Module}

The LMS module is the module that acts as an intermediary between learners and systems. As parts of the LMS module are connected to most of the other modules in order to use it in learning and teaching.

\section{Adaptive Module}

The adaptive module is the module that improves learning. The module's function is to monitor learner's learning status 
with testing between classes passing on criteria test scores. Adaptive module will encourage learners to study other chapters further, but if the test scores between classes do not meet the criteria, this module will suggest returning to review again, etc.

\section{E. Learning Content Module}

The Learning Content Module is the module that stores learning contents which comes from the analysis of 9 Multiple Intelligences and divided into 3 groups; 1) Analytic content used with learners who have analytical and mathematical aptitude. 2) Introspective content used with learners who have the imaginative and artistic aptitude. 3) Interactive content used with learners who have the skills of communication and interactive aptitude to others.

\section{DESIGN OF RUle BASE}

\section{A. Acknowledge Process for Rule base Design}

The process of creating a rule base consists of 4 steps. 1) First step is a survey of variables that affects the ability of Multiple Intelligence for students. This process studies previous research to study variables that have an impact on the ability of Multiple Intelligences. Moreover, the author of this paper also interviewed Multiple Intelligence experts. 2) The second step is to create the questionnaires to survey data from sample groups. The questions of the questionnaires consist of two parts. One covering general information about the respondents (defined as a main variable) such as name, year, field, faculty, etc. The second part is the question to separate the ability of Multiple Intelligences (defined as a prediction variable). 3) The third step is an information survey from a sample of students to answer the questionnaires created from the last part. This paper uses the sample of 3,000 TNI students. 4) The fourth step analyzes the survey information from the third step using Data Mining.

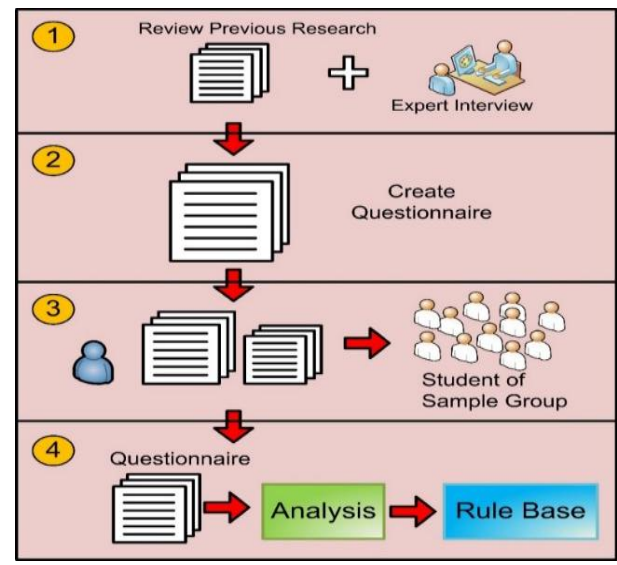

Fig. 3. Process of Rule base creation.

This research uses five algorithms for analysis. 1) ID3 algorithm 2) C4.5 algorithm 3) NBTree algorithm 4) Naïve Bayes algorithm and 5) Bayes Net algorithm. Process for selection algorithm. Then, results from the 5 algorithms will be compared to find the most efficient referring to prediction. Fig. 3 shows the process of rule base design.

\section{B. Comparison of Algorithm}

This section presents the evaluation of creating a rule base to guide students by considering the percentage of precision from student's ability. One can compare the evaluation of the rule base by using 5 methods; 1) ID3 algorithm 2) C4.5 algorithm 3) NBTree algorithm 4) Naïve Bayes algorithm and 5) Bayes Net algorithm. The results of the comparison are shown in Table I.

TABLE I: COMPARISON OF ALGORITHM FOR RULE BASE

\begin{tabular}{|l|l|}
\hline Algorithm & $\%$ Prediction \\
\hline 1. ID3 Algorithm & 78.615 \\
\hline 2. C4.5 Algorithm & 83.436 \\
\hline 3. NBTree Algorithm & 77.008 \\
\hline 4. Naïve Bayes Algorithm & 60.939 \\
\hline 5. Bayes Net Algorithm & 66.378 \\
\hline
\end{tabular}

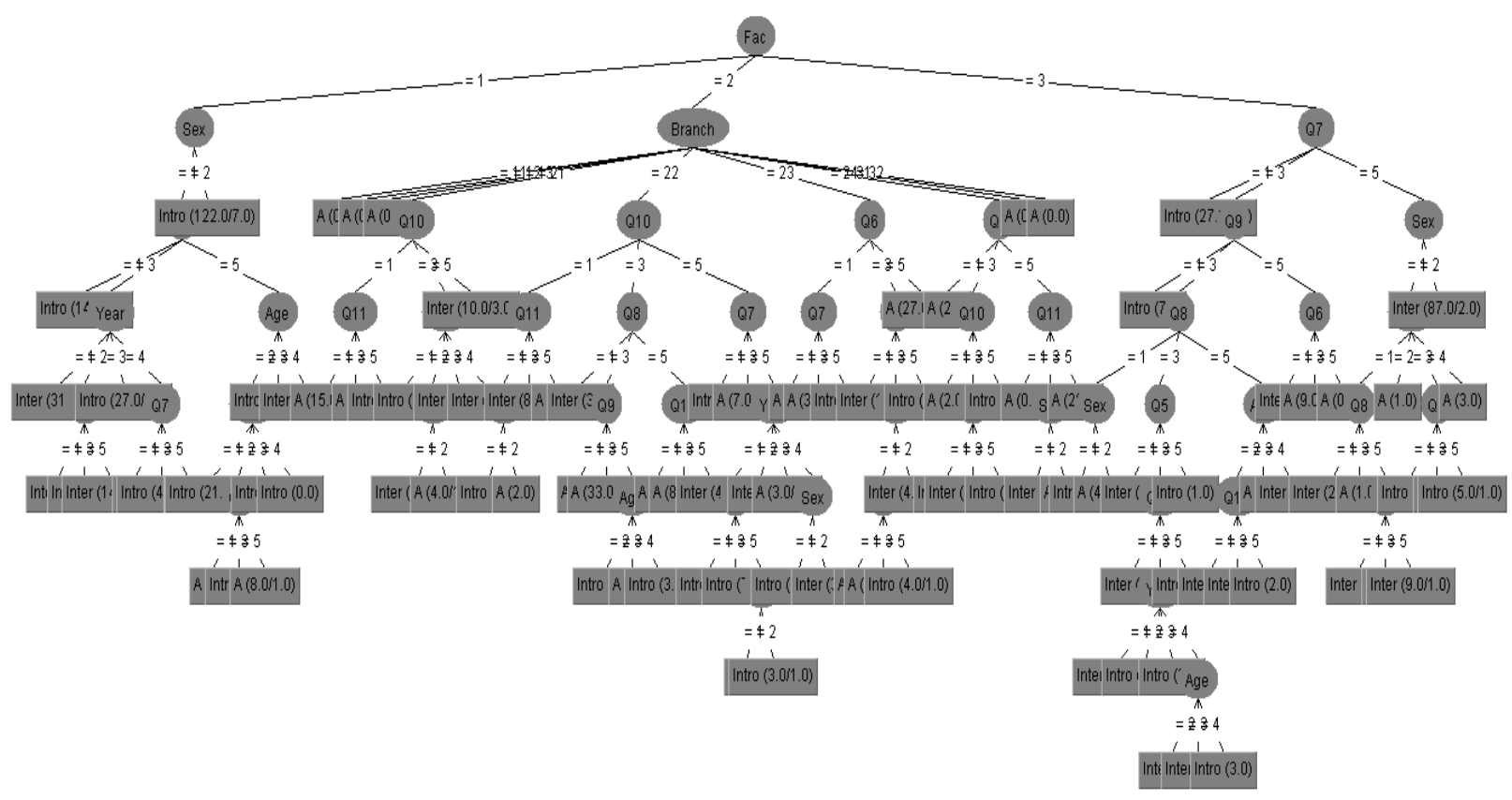

Fig. 4. Result of C4.5 algorithm rule base. 


\begin{tabular}{|c|c|c|}
\hline No & Rule Base & Prediction \\
\hline 1 & IF Fac $=1$ and $S e x=1$ and $Q 5=$ Low & Intro \\
\hline 2 & IF Fac $=1$ and $S e x=1$ and $Q 5=$ Medium and $Y e a r=1$ & Intro \\
\hline 3 & IF Fac $=1$ and $S e x=1$ and $Q 5=$ Medium and $Y e a r=2$ and $Q 7=$ Low & Intro \\
\hline 4 & IF Fac $=1$ and $S e x=1$ and $Q 5=$ Medium and $Y e a r=2$ and $Q 7=$ Medium & Intro \\
\hline 5 & IF Fac $=1$ and $S e x=1$ and $Q 5=$ Medium and Year $=2$ and $Q 7=$ High & Inter \\
\hline 6 & IF Fac $=1$ and $S e x=1$ and $Q 5=$ Medium and $Y e a r=3$ & Intro \\
\hline 7 & IF Fac $=1$ and $S e x=1$ and $Q 5=$ Medium and $Y e a r=4$ and $Q 7=$ Low & Analytic \\
\hline 8 & IF Fac $=1$ and $S e x=1$ and $Q 5=$ Medium and Year=4 and Q7=Medium & Intro \\
\hline 9 & IF Fac $=1$ and Sex $=1$ and Q5=Medium and Year=4 and Q7=High & Analytic \\
\hline 10 & IF Fac $=1$ and $S e x=1$ and $Q 5=$ High and Age $=1$ and $Y e a r=1$ & Intro \\
\hline 11 & IF Fac $=1$ and $S e x=1$ and $Q 5=$ High and $A g e=1$ and $Y e a r=2$ and Q7=Low & Analytic \\
\hline 12 & IF Fac $=1$ and $S e x=1$ and $Q 5=$ High and $A g e=1$ and $Y e a r=2$ and $Q 7=$ Medium & Intro \\
\hline 13 & IF Fac $=1$ and $S e x=1$ and $Q 5=\mathrm{High}$ and $\mathrm{Age}=1$ and $Y$ ear $=2$ and Q7=High & Analytic \\
\hline 14 & IF Fac $=1$ and $S e x=1$ and $Q 5=$ High and $A g e=1$ and $Y e a r=3$ & Intro \\
\hline 15 & IF Fac $=1$ and $S e x=1$ and $Q 5=$ High and Age $=1$ and Year $=4$ & Intro \\
\hline 16 & If Fac $=1$ and $S e x=1$ and $Q 5=$ High and $A g e=2$ & Intro \\
\hline 17 & IF Fac $=1$ and $\mathrm{Sex}=1$ and $Q 5=\mathrm{High}$ and $\mathrm{Age}=3$ & Inter \\
\hline 18 & IF Fac $=1$ and $S e x=2$ & Intro \\
\hline 19 & IF Fac $=2$ and $\mathrm{Branch}=11$ & Analytic \\
\hline 20 & IF Fac $=2$ and $\mathrm{Branch}=12$ & Analytic \\
\hline
\end{tabular}

Fig. 5. Example of rule base.

Table I shows the comparison of the rule base creation. The result of each algorithm follows, ID3 algorithm equaled $78.615 \%$, C4.5 algorithm equaled $83.436 \%$, NBTree algorithm equaled $77.008 \%$, Naïve Bayes algorithm equaled $60.939 \%$, Bayes Net algorithm equaled $66.378 \%$. This paper focused on percentage of prediction for each algorithm. C4.5 algorithm was found to have the highest percentage of prediction. Percentage of prediction of $\mathrm{C} 4.5$ algorithm equaled $83.436 \%$.

\section{An Example of the Rule Base}

Result of the rule base design for C4.5 algorithm is depicted in Fig. 4. An example of the rule base is depicted in Fig. 5.

\section{CONCLUSION}

The objective of this research was to design a rule base to recommend e-learning and learning activities based on Multiple Intelligence. Design of the rule base consists of four parts. The first part was a survey of the variables. Second part was creation of the questionnaires. Third part was a survey using student sample groups. The fourth part was an analysis of data which came from the results of the survey. The process for selection of the rule base was undertaken by comparing 5 algorithms as follows 1) ID3 algorithm 2) C4.5 algorithm 3) NBTree algorithm 4) Naïve Bayes algorithm 5) Bayes Net algorithm. The result of each algorithm is, ID3 algorithm are as follows $78.615 \%$, C4.5 algorithm equaled $83.436 \%$, NBTree algorithm equaled $77.008 \%$, Naïve Bayes algorithm equaled $60.939 \%$, Bayes Net algorithm equaled $66.378 \%$. When considering percentage of prediction for each algorithm, C4.5 algorithm had the highest percentage of prediction. Percentage of prediction for the C4.5 algorithm equaled $83.436 \%$.

Alongside the rule base recommendation system the author of this paper also proposed a model of recommendation for e-learning and learning activities. The conceptual model was divided into 4 sections; 1) The Rule base section separates the form of students' learning into 3 patterns from the aptitude of
Multiple Intelligences. 2) The Recommendation module introduces students to a detailed content which matched their aptitude. This module will match rules from the Adaptive module. 3) The LMS module for learning and teaching. 4) The e-Mentor module automatically sents the instructions to the students who needed the assistance. 5) Learning content module stored contents of Multiple Intelligences approaches which consisted of three types 1) Analytic content 2) Introspective content 3) Interactive content.

\section{REFERENCES}

[1] E-learning System. (2014). [Online]. Available: http://en.wikipedia.org/wiki/E-learning

[2] Distance Learning. (2014). [Online]. Available: http://en.wikipedia.org/wiki/Distance_education

[3] Self Learning. (2014). [Online]. Available: http://en.wikipedia.org/wiki/Self-regulated_learning

[4] T. Yuan and C. Chen, "Semantic ideation learning for agent-based e-brainstorming," IEEE Trans, vol. 20, no. 2, pp. 261-275, 2008.

[5] P. Polkacha and M. Tiantong, "A conceptual framework of an online project based learning model with intelligence knowledge management and using KWL plus for assessment," in Proc. NCCIT, Bangkok, pp. 734-739, 2011.

[6] F. Aruan, S. Prihatmanto, and H. Kuspriyanto, "The designing and implementation of a problem based learning in collaborative virtual environments using MMOG technology," in Proc. ICSET/IEEE, Bandung, pp. 1-7, 2012.

[7] S. Teemuangsai, "The development of a model of collaborative problem-based learning with scaffolding via computer network," Ph. D. dissertation, Dept. Com. Ed., Kmutnb Univ., Bangkok, Thailand, 2009.

[8] S. Saiboonruen, "A conceptual framework of an online simulation problem based learning with scaffolding system," in Proc. NCCIT, Bangkok, pp. 656-661, 2011.

[9] K. Duangkaew and S. Tangwannawit, "Adaptive presentation of activities learning system based on multiple intelligences," in Proc. NCCIT, Bangkok, pp. 343-348, 2011.

[10] S. Tangwannawit, N. Sureerattanan, and M. Tiantong, "Multiple intelligences learning activities model in e-Learning environment," Special Issue of the International Journal of the Computer, the Internet and Management, vol. 16, pp. 25.1-25.4, 2008.

[11] Student-centered learning System. (2014). [Online]. Available: http://www.en.wikipedia.org/wiki/student-center_learning

[12] S. Nattakorn, "A development of computer game for enhancing agricultural comprehension on Chinese cultivation," Journal of King Mongkut Institute of Technology Ladkrabang, vol. 2, pp. 1-15, 2006.

[13] T. Kaewkiriya, "A design and development of e-learning content for multimedia technology using multimedia game," International 
Journal of Software Engineering and Application, vol. 4, no. 6, pp. 61-69, 2013

[14] O. Pantho and M. Tiantong, "Conceptual framework of synthesize on an adaptive e-learning with e-mentoring system using VARK learning style by data mining methodology," International Journal of Computer Technology.

[15] N. D. Fleming, "I'm different; not dumb. Modes of presentation (VARK) in the tertiary classroom," in Proc. Annual Conference of the Higher Education and Research Development Society of Australasia (HERDSA), pp. 308-313, 1995.

[16] B. Gardner and S. Korth, "A framework for learning to work in teams," Journal of Education for Business, vol. 74, no. 1, pp. 28-33, 2010.

[17] S. Tangwannawit, "Learning activities model in e-learning environment with the development of different multiple intelligences learners," Ph.D. dissertation, Dept. Com. Ed., Kmutnb Univ, Bangkok, Thailand, 2010.

[18] A. Firte, "Intelligent component for adaptive e-learning systems," in Proc. IEEE 5th International Conference on Intelligent Computer Communication and Processing, pp. 35-38, 2009.

[19] N. Norwawi, "Classification of students' performance in computer programming course according to learning style," IEEE-DMO'09, pp. $37-41,2009$.

[20] S. V. Kolekar et al., "Learning style recognition using artificial neural network for adaptive user interface in e-learning," in Proc. IEEE-Computational Intelligence and Computing Research, pp. 1-5, 2010.

[21] T. Kaewkiriya, N. Utrakrit, S. Tangwannawit, and M. Tiantong, "A conceptual framework of synthesize on an adaptive e-Learning guidance system base on multiple intelligence," International Journal of Information and Electronic Engineering, vol. 3, no. 6, pp. 622-625, 2013.

[22] S. Chen, B. Mulgrew, and P. M. Grant, "A clustering technique for digital communications channel equalization using radial basis function networks," IEEE Trans. on Neural Networks, vol. 4, pp. 570-578, July 1993.

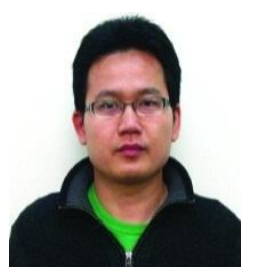

Thongchai Kaewkiriya graduated with a bachelor degree in computer technology and electronic telecommunication engineering from King Mongkut's University of Technology North Bangkok, Thailand in 2000 and Pathumwan Institute of Technology Bangkok, Thailand in 2006.

He graduated with a master degree in electrical and information engineering from King Mongkut's University of Technology Thonburi, Thailand in 2005. He graduated with a doctor degree in electrical engineering and information science from Osaka Prefecture University, Japan in 2014. He works as a lecturer in the field of information technology while also looking after the Information and Communication Center at Thai-Nichi Institute of Technology, Thailand.

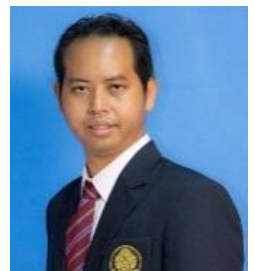

Nattavee Utrakit received a doctoral degree from Edith Cowan University (ECU), Australia, in 2006 in information technology. He works as a lecturer and a researcher at the Department of Information Technology Management, Faculty of Information Technology, King Mongkut's University of Technology North Bangkok (Kmutnb), Thailand. He is also a member of the Applied Information Systems Research Division (AIS) at Kmutnb.

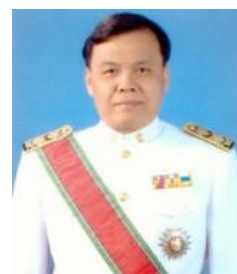

Monchai Tiantong is a TechEd.D. He received his B.S. (electrical engineering), M.S. (electrical), and TechEd.D. (curriculum research \& development) from King Mongkut's University of Technology North Bangkok (Kmutnb), Bangkok, Thailand. He has been working as an associate professor for more than 20 years in the field of information technology and computer education at the Faculty of Technical Education, Kmutnb. He is also a member of AACE, IEEE, ACM, etc 